\title{
Lixiviação do imazethapyr em solo de várzea sob dois sistemas de manejo
}

\author{
Imazethapyr leaching in rice paddy soil under two tillage systems
}

\author{
Alejandro Fausto Kraemer ${ }^{\mathrm{I}}$ Enio Marchesan ${ }^{\text {II }}$ Mara Grohs $^{\text {III }}$ Luis Antonio de AvilaII \\ Sérgio Luiz de Oliveira Machado ${ }^{\mathrm{IV}}$ Renato Zanellav ${ }^{\mathrm{V}}$ Paulo Fabricio Sachet Massoni ${ }^{\mathrm{II}}$ \\ Gerson Meneghetti Sarzi SartoriII
}

\section{RESUMO}

O imazethapyr está sendo amplamente utilizado no Rio Grande do Sul desde o desenvolvimento da Tecnologia Clearfield ${ }^{\circledast}$ (CL) em arroz, em uma mistura formulada de imazethapyr + imazapic $\left(75+25 \mathrm{~g} \mathrm{~L}^{-1}\right)$. No entanto, com o uso dessa tecnologia, surgiu o problema da persistência do herbicida no solo, afetando cultivos não tolerantes em sucessão com diferentes intensidades. A fitointoxicação está relacionada, dentre vários fatores, à localização do herbicida no perfil do solo. O presente trabalho teve por objetivo determinar o posicionamento do imazethapyr em profundidade, no perfil de um solo de várzea cultivado com arroz, frente a dois tipos de manejo. Foram coletadas amostras de solos, em diferentes profundidades (0-5, 5-10, 10-15 e 15-20cm), do solo de várzea sob dois sistemas de manejo: plantio convencional $(P C) e$ plantio direto (PD), em uma área onde havia sido utilizado arroz CL por dois anos e no terceiro ano, arroz não tolerante. A concentração do herbicida no solo foi analisada por HPLC$D A D$. Há maior concentração de imazethapyr na superfície do solo $(0-5 \mathrm{~cm})$, no sistema convencional, quando comparado com o sistema de plantio direto, e o herbicida lixiviou até $20 \mathrm{~cm}$ nos dois sistemas. No PC, ocorreu uma distribuição uniforme do imazethapyr nos primeiros $15 \mathrm{~cm}$ de solo, enquanto que, no $P D$, constatou-se acúmulo de imazethapyr nas camadas de 5-10 e 10-15cm.

Palavras-chave: percolação de herbicidas, residual, preparo de solo, HPLC, Oryza sativa.

\begin{abstract}
Imazethapyr has been widely used in Rio Grande do Sul since the development of Clearfield Technology ${ }^{\mathrm{TM}}$ (CL) on rice, in a formulated mixture of imazethapyr + imazapic $\left(75+25 \mathrm{~g} \mathrm{~L}^{-1}\right)$. However, the use of such technology raised the problem of herbicide carryover, which might affect non-tolerant crops in rotation with different intensities. The plant injury is related, among other factors, with the herbicide position in the soil profile. The present work had the objective of determining the depth positioning of imazethapyr on a lowland soil cultivated with rice in two soil tillage system: conventional system (CS) and no till system (NT), in an area where CL rice had been cultivated for two years followed by non tolerant rice in the third year. Herbicide concentration in soil samples was analyzed by HPLC-DAD. There is a higher concentration of imazethapyr in the topsoil $(0-5 \mathrm{~cm})$ on the $C S$, when compared to the NT, while it leached until $20 \mathrm{~cm}$ in both systems. In the CS, imazethapyr were uniformly distributed in the first $15 \mathrm{~cm}$ of soil, whereas in NT, imazethapyr were accumulated in 5-10 and $10-15 \mathrm{~cm}$ layers.
\end{abstract}

Key words: herbicide percolation, residual, soil plowing, HPLC, Oryza sativa.

\section{INTRODUÇÃO}

O herbicida imazethapyr pertence à família das imidazolinonas e controla um amplo espectro de

IPrograma de Pós-graduação em Agronomia, Universidade Federal de Santa Maria (UFSM). Instituto Nacional de Tecnología Agropecuaria (INTA), Corrientes, República Argentina.

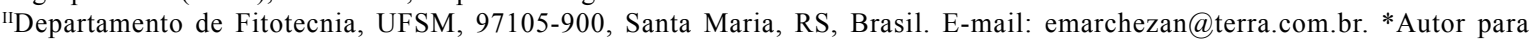
correspondência.

IIICurso de Agronomia, UFSM, Santa Maria, RS, Brasil.

IV Departamento de Defesa Fitossanitária, UFSM, Santa Maria, RS, Brasil.

vepartamento de Química, UFSM, Santa Maria, RS, Brasil. 
plantas daninhas, incluindo gramíneas, ciperáceas e latifoliadas. É absorvido pelas raízes e folhas (TAN et al., 2005) e transportado pelo floema e xilema, acumulando-se nos pontos de crescimento. $\mathrm{O}$ controle é proporcionado pela inibição da enzima acetolactato sintetase (ALS), que é essencial no processo de síntese de aminoácidos de cadeias ramificadas em plantas, como a valina, leucina e isoleucina (TAN et al., 2006). Esse herbicida está sendo amplamente utilizado no Estado do Rio Grande do Sul desde o desenvolvimento da Tecnologia Clearfield ${ }^{\circledR}$ em arroz, em uma mistura formulada de imazethapyr + imazapic $\left(75+25 \mathrm{~g} \mathrm{~L}^{-1}\right)$, com o nome comercial de Only ${ }^{\circledR}$.

Imazethapyr apresenta a característica de ser persistente no solo, sendo muito solúvel em água e suscetível à lixiviação (BATTAGLIN et al., 2000; MADANI et al., 2003). A localização do herbicida na zona de absorção das raízes pode comprometer a utilização futura da área com culturas não tolerantes. Há relatos e experiências indicando fitointoxicação causada pelo residual do imazethapyr, que são variáveis, dependendo das condições físicas, químicas e de manejo do solo, dentre outros fatores. MASSONI et al. (2007) relataram efeito residual desse herbicida sobre o arroz suscetível 358 dias após a aplicação. Para WILLIAMS et al. (2002), devem transcorrer 540 dias (18 meses) entre a aplicação de imazethapyr e a semeadura de arroz não tolerante.

A persistência do imazethapyr no solo depende das condições climáticas, das propriedades do solo e da dose do herbicida. O principal mecanismo de dissipação do imazethapyr no solo é a degradação microbiana aeróbica, sendo a degradação anaeróbica praticamente inexistente (SHANER \& O`CONNOR, 1991). Outra forma de saída do herbicida da zona de absorção das raízes, que não envolve degradação, é a lixiviação. Para o imazapyr, a quantidade do herbicida que lixivia está mais relacionada com a permeabilidade do solo, sendo maior em solos bem estruturados e arenosos do que em solos argilosos e com maior densidade (ONA et al., 2007). A sorção também exerce efeito sobre a lixiviação, e quanto maior a sorção menor a lixiviação. Por sua vez, a sorção é inversamente correlacionada ao $\mathrm{pH}$ e diretamente correlacionada ao teor de matéria orgânica do solo (BÖRJESSON et al., 2004).

O movimento de imazethapyr em profundidade é maior quanto maior a quantidade de chuva, sendo importante no processo de lixiviação. Segundo McDOWELL et al. (1997), em condições de baixa precipitação, imazapyr concentrou-se na camada superficial, diminuindo drasticamente sua concentração abaixo de $10 \mathrm{~cm}$. Com altas precipitações, o herbicida atingiu maior profundidade, distribuindo-se mais uniformemente nos primeiros $15 \mathrm{~cm}$ de solo. Determinações de JOURDAN et al. (1998) mostraram que imazethapyr movimentou-se em profundidade em um solo arenoso, conforme transcorreram os dias após a aplicação (DAA) do herbicida. Nos primeiros cinco DAA, o herbicida atingiu $20 \mathrm{~cm}$, embora as maiores concentrações se encontrassem nos primeiros $10 \mathrm{~cm}$. A maior concentração do produto, aos 30DAA, foi detectada nos primeiros $15 \mathrm{~cm}$, embora tenha ocorrido até $30 \mathrm{~cm}$ de profundidade. A partir dos 90DAA, a concentração, nos primeiros cinco centímetros, diminuiu, concentrando-se o herbicida entre 5 e $30 \mathrm{~cm}$ de profundidade. De acordo com HOLLAWAY et al. (2006), o imazethapyr pode permanecer no solo por mais de três anos e alcançar até $40 \mathrm{~cm}$ de profundidade.

O imazethapyr e imazapyr lixiviam rapidamente após uma chuva; porém, durante o processo natural de perda de umidade do solo, o herbicida movimenta-se para cima, conduzido pelas correntes de evapotranspiração (VAN WYK \& REINHARDT, 2001; FIRMINO et al., 2008). Esses movimentos são mais pronunciados em pHs mais elevados, e a mobilidade desse herbicida no perfil do solo decresce com o transcorrer do tempo de aplicação do produto (JOHNSON et al., 2000).

O conhecimento da profundidade de acúmulo do herbicida possibilita manejar a profundidade de semeadura de cultivos não tolerantes, como alternativa para diminuir o efeito prejudicial do herbicida. $\mathrm{O}$ posicionamento das raízes ou de outros órgãos de absorção (coleóptilo) fora da região de maior concentração de um herbicida é um dos mecanismos de seletividade. $\mathrm{O}$ arroz semeado mais profundo desenvolve coleóptilos mais compridos e um sistema radicular mais profundo, estando exposto à absorção de maior quantidade de herbicida com alta mobilidade no solo, como o imazethapyr (ZHANG et al., 2000). Em sorgo, a fitointoxicação por metholachlor aumenta na medida em que aumenta a profundidade de semeadura.

Em vista do exposto, o presente trabalho teve por objetivo determinar o efeito de dois sistemas de manejo do solo nas concentrações e no posicionamento do imazethapyr em profundidade, nas áreas de várzea cultivadas com arroz.

\section{MATERIAL E MÉTODOS}

O experimento foi conduzido em solo de várzea, classificado como Planossolo Hidromórfico eutrófico arênico, com as seguintes características: $\mathrm{pH}_{\text {áqua }}(1: 1)=4,8 ; \operatorname{argila}=26 \% ;$ M.O. $=23 \mathrm{~g} \mathrm{~kg}^{-1} ; \mathrm{P}=6,0 \mathrm{mg}$ $\mathrm{dm}^{-3} ; \mathrm{K}=120 \mathrm{mg} \mathrm{dm}^{-3} ; \mathrm{Ca}=5,0 \mathrm{cmolc} \mathrm{dm}^{-3} ; \mathrm{Mg}=2,0 \mathrm{cmolc}$ 


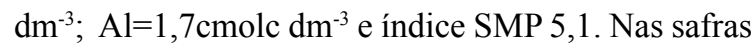
agrícolas 2004/05 e 2005/06, a área foi cultivada com arroz Clearfield (CL) e, na safra agrícola 2006/07, com arroz não tolerante (NT). Nas safras com arroz CL, foi aplicado imazethapyr+imazapic $\left(\mathrm{Only}^{\oplus}\right)$ na dose de $75+25 \mathrm{~g} \mathrm{~L}^{-1}$, respectivamente. Antes da semeadura do arroz NT, foram adotados dois sistemas de manejo de solo, plantio direito (PD), sem movimentação de solo, e plantio convencional (PC), com preparos de solo em abril, maio, agosto e outubro. Durante o mesmo período, foi medida a profundidade do lençol freático uma vez por semana. Para tanto, foram construídos dois poços de observação por bloco com cano de PVC perfurado de $90 \mathrm{~cm}$ de profundidade e $5 \mathrm{~cm}$ de diâmetro. No mesmo período, foram realizadas duas medições diárias de temperatura de solo a $3 \mathrm{~cm}$ de profundidade, às nove $\mathrm{e}$ às 15 horas, com as quais foi calculada a temperatura média diária do solo a $3 \mathrm{~cm}$, para os dois sistemas de preparo de solo avaliados.

Após a colheita do arroz NT (540 dias após a última aplicação do herbicida), foram coletadas, em cada parcela, amostras de solo a quatro profundidades, de $0-5,5-10,10-15$ e $15-20 \mathrm{~cm}$. As amostras foram compostas por três subamostras recolhidas em diferentes locais de cada parcela. Logo após coletadas, as amostras de solo foram secas ao ar e na sombra e posteriormente foram moídas e conservadas em freezer até serem feitas as análises de laboratório. A extração de imazethapyr do solo foi realizada utilizando-se metodologia descrita por GONÇALVES (2003), e a quantificação do herbicida foi realizada utilizando-se cromatografia líquida de alta eficiência com detecção por arranjo de diodos (HPLC-DAD). Para obtenção da quantidade total de imazethapyr por metro quadrado, a concentração do herbicida, em cada profundidade, foi convertida para quantidade por área, sendo corrigidos os valores pela densidade do solo em cada profundidade (VIZZOTTO et al., 2000).

Foram estudados oito tratamentos oriundos da combinação de dois manejos de solo (PD e PC) com as quatro profundidades de amostragem (0-5, 5-10, 1015 e $15-20 \mathrm{~cm})$. O delineamento experimental foi de blocos ao acaso, com quatro repetições, em arranjo fatorial. Os dados foram submetidos à análise de variância, e as médias foram comparadas pelo teste de Tukey, a $5 \%$ de probabilidade de erro. Para a análise estatística, os dados foram transformados em yt $=\sqrt{\mathrm{y}+0,5}$.

\section{RESULTADOS E DISCUSSÃO}

Comparando-se a quantidade total de imazethapyr presente no solo (somatório das quantidades do herbicida encontrado de $0-20 \mathrm{~cm}$ )
(Figura 1), observa-se que não houve diferença na quantidade encontrada no PD $\left(1343 \mu \mathrm{g} \mathrm{m}^{-2}\right.$, o equivalente a $13 \mathrm{~g}$ de imazethapyr ha $\mathrm{a}^{-1}$ ) em relação ao PC ( $1281 \mu \mathrm{g} \mathrm{m}^{-2}$, o que equivale a $12 \mathrm{~g}$ de imazethapyr $\mathrm{ha}^{-1}$ ), indicando que não há diferença na degradação do herbicida entre os sistemas no somatório das profundidades. Esses resultados contrastam com os de CURRAN et al. (1992), os quais observaram que a fitointoxicação de imazaquin e imazethapyr em milho foi maior no sistema de PD do que no PC. RENNER et al. (1998), comparando o efeito de diferentes sistemas de preparo de solo na dissipação do imazaquin, encontraram que, no preparo de solo com arado, o herbicida foi degradado mais rapidamente que no preparo com escarificador e este, por sua vez, mais que no PD. Trabalho de ULBRICH et al. (2005) relata menor degradação de imazapic e imazapyr em dois solos com PD, em comparação com o solo com PC. Comparando preparos de solo com diferentes equipamentos, SEIFERT et al. (2001) não encontraram diferenças na degradação do imazaquin entre o preparo com arado e escarificador. MONKS \& BANKS (1993) mostraram que não houve efeito de diferentes manejos de palha e de preparo de solo no residual de imazaquin e imazethapyr.

A diferença de fitotoxicidade entre os preparos de solo pode estar relacionada ao posicionamento do herbicida no solo e não à quantidade total de herbicidas por área. Com relação às profundidades de localização do herbicida (Figura 2), observou-se interação significativa entre os sistemas de manejo de solo e as profundidades, por isso o comportamento do imazethapyr foi analisado comparando-se as profundidades dentro de cada manejo de solo. Imazethapyr lixiviou até $20 \mathrm{~cm}$ de profundidade independentemente do sistema de manejo utilizado, embora a concentração detectada nessa profundidade seja pequena $\left(0,8 \mu \mathrm{g} \mathrm{kg}^{-1}\right)$. Resultados similares foram encontrados por RENNER et al. (1998) com imazaquin, que foi detectado até $18 \mathrm{~cm}$ de profundidade independentemente do sistema de preparo de solo empregado (arado escarificador e PD). Resultados de outros trabalhos indicam que o imazethapyr alcançou $30 \mathrm{~cm}$ de profundidade em solos com baixos teores de argila, três meses após a aplicação do herbicida (McDOWELL et al., 1997; JOURDAN et al., 1998; VAN WYK \& REINHARDT, 2001).

No PC, o herbicida distribuiu-se uniformemente nas quatro camadas de solo avaliadas. No entanto, no PD, observou-se menor concentração na camada de $0-5 \mathrm{~cm}$, quando comparado com o sistema convencional, com concentração crescente com o aumento da profundidade até $15 \mathrm{~cm}$, e estas diminuíram drasticamente de $15-20 \mathrm{~cm}$, para ambos os sistemas. A 


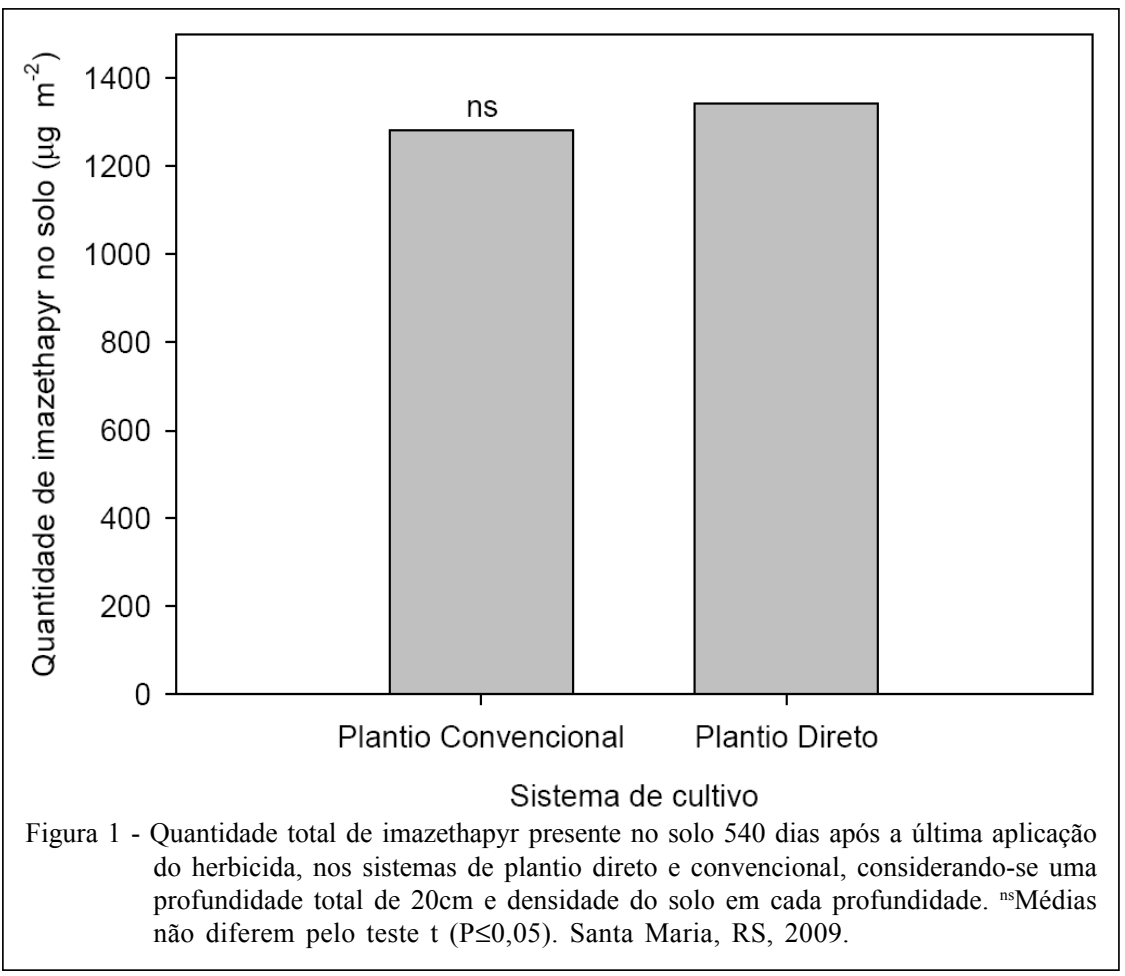

distribuição uniforme, nas três camadas superficiais de solo (primeiros $15 \mathrm{~cm}$ ), no PC, possivelmente esteja relacionada com uma maior evapotranspiração da água do solo nesse sistema, quando comparado com o PD.
A temperatura média do solo na superfície do solo do PC foi maior que do PD (Figura 3), o que poderia ter gerado maior evaporação e corrente ascendente de água, arrastando consigo o herbicida para a camada

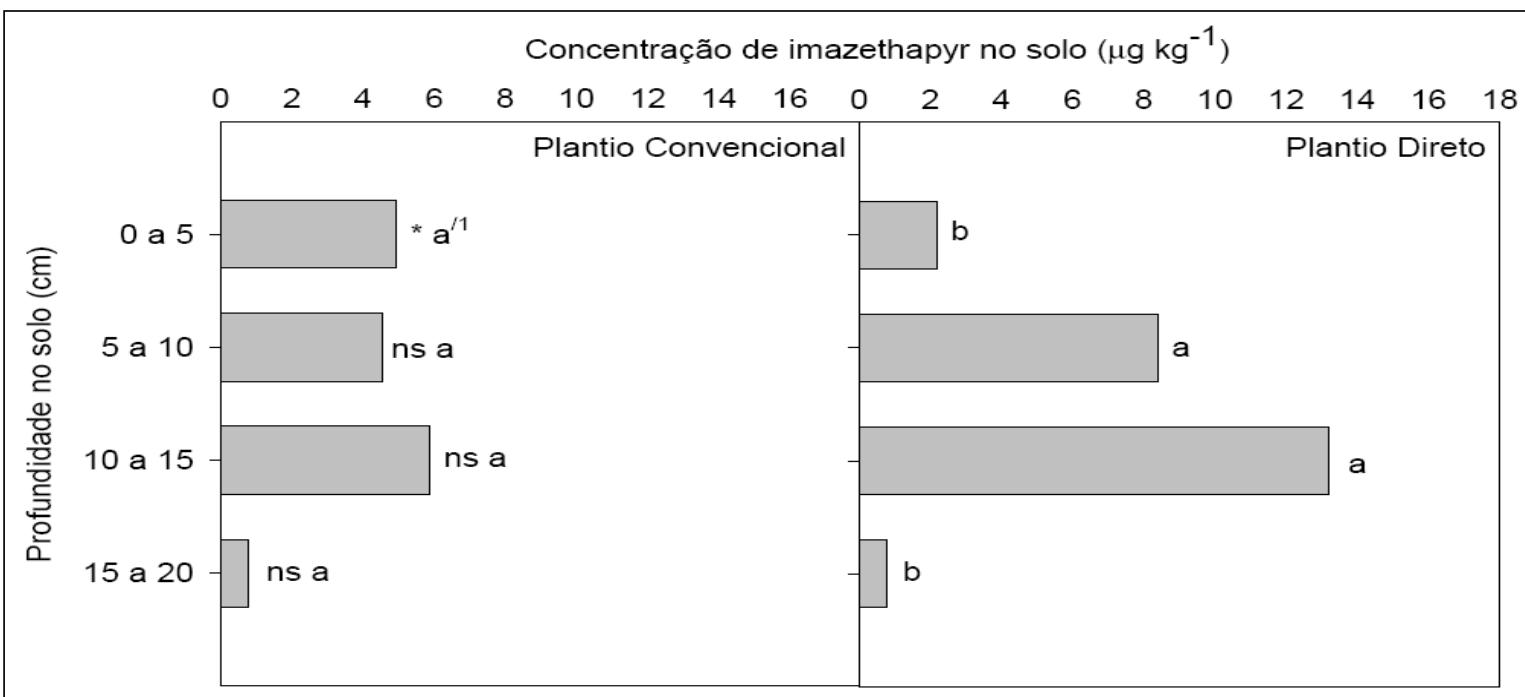

Figura 2 - Concentração de imazethapyr, expressa em $\mu \mathrm{g}$ de ingrediente ativo por $\mathrm{kg}$ de solo $\left(\mu \mathrm{g} \mathrm{kg} \mathrm{kg}^{-1}\right)$, em dois manejos de solo, plantio convencional (PC) e plantio direto (PD) e quatro profundidades $(0-5,5-10 ; 10-15$ e $15-20 \mathrm{~cm})$, após a colheita de arroz no terceiro ano de um sistema de rotação com dois anos de arroz Clearfield ${ }^{\circledR}$ e um ano de arroz não tolerante (540 dias após a última aplicação do herbicida). Santa Maria, RS, 2009. ${ }^{1 /}$ Médias não ligadas por mesma letra minúsculas, comparando profundidades, diferem entre si pelo teste de Tukey, a 5\% de probabilidade de erro; ${ }^{\text {ns Não há }}$ diferença significativa entre os sistemas de preparo do solo; *Diferença significativa entre os sistemas de preparo do solo. 


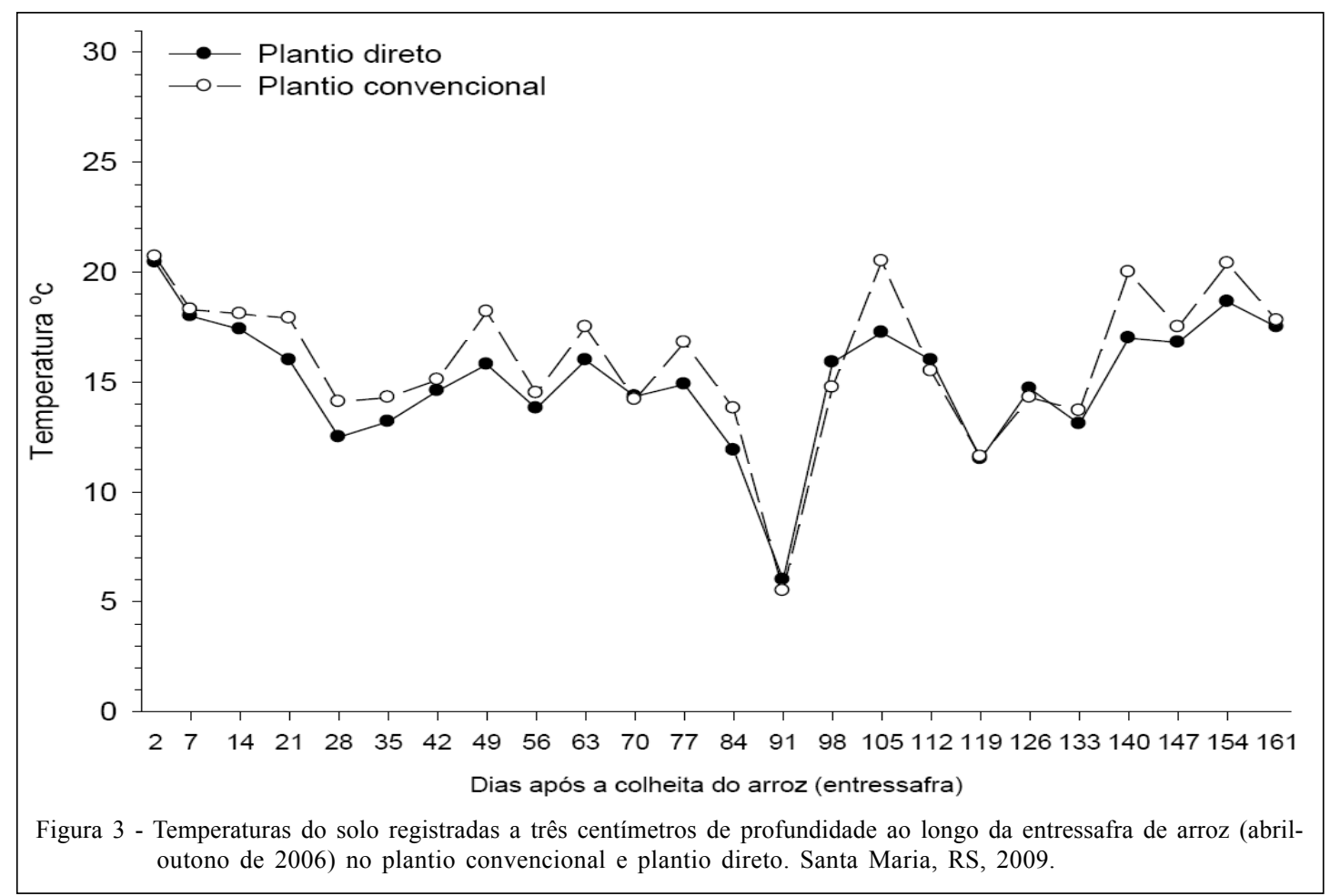

superficial, colocando-o em uma região de maior concentração de raízes e possibilitando, assim, maior fitotoxicidade em plantas não tolerantes no PC. Essa hipótese encontra respaldo em resultados de VAN WYK \& REINHARDT (2001) e FIRMINO et al. (2008), os quais relatam que imazethapyr e imazapyr lixiviam rapidamente para as camadas subsuperficiais, mas que, com as correntes geradas pela evapotranspiração, são transportados novamente para a superfície.

A maior concentração de imazethapyr, em profundidades maiores no solo $(5-15 \mathrm{~cm})$, provavelmente deve-se à permanência desse herbicida nessas profundidades, onde as condições são desfavoráveis a sua degradação. As condições ambientais, como temperatura média mais baixa, conforme discutido anteriormente, menor aeração do solo e menor disponibilidade de nutrientes pela não incorporação da matéria orgânica, podem ter desfavorecido o desenvolvimento dos microorganismos aeróbicos, reduzindo assim a velocidade de degradação do herbicida. Nesse sentido, Franchini et al. (2007) observaram que a atividade microbiana incrementa, em média, $57 \%$ após um preparo do solo como consequência da incorporação da matéria orgânica e da maior aeração. A temperatura do solo também afeta a degradação das imidazolinonas. Em solos cultivados sob temperaturas de 18 e $35^{\circ} \mathrm{C}$, a degradação do imazethapyr foi de 66 e 100\%, respectivamente (BASHAM \& LAVY, 1987). Além disso, FLINT \& WITT (1997) determinaram que a emissão de $\mathrm{CO}_{2}$ por micro-organismos aumenta com a presença de imazethapyr ou imazaquin, duplicando a atividade quando a temperatura passa de 15 para $30^{\circ} \mathrm{C}$. Com esse aumento, observou-se redução na concentração de imazethapyr, que foi mais acentuada em solo úmido (JOURDAN et al., 1998). Autores como BASHAM \& LAVY (1987) e BAUGHMAN \& SHAW (1996) demonstraram que imazethapyr e imazaquin foram mais persistentes em solos com temperaturas mais baixas e reduzido teor de umidade.

Dessa forma, as condições foram mais desfavoráveis à atividade microbiológica em profundidade no sistema PD, colaborando, possivelmente, com a menor degradação do herbicida nessas profundidades. Além disso, no PD, o imazethapyr acumulou-se entre 5 e $15 \mathrm{~cm}$, e durante a entressafra o lençol freático esteve acima de $20 \mathrm{~cm}$ de profundidade (Figura 4) por nove semanas, deixando essa região em anaerobiose por prolongados períodos de tempo, o que reduz a degradação do herbicida. Essa afirmação é baseada no fato que a principal via de degradação do imazethapyr é a degradação microbiana (FLINT \& WITT, 1997) promovida por microorganismos aeróbicos, sendo praticamente inexistente em anaerobiose (SHANER \& O`CONNOR, 1991). Por sua vez, isso estaria contribuindo para a menor

Ciência Rural, v.39, n.6, set, 2009. 


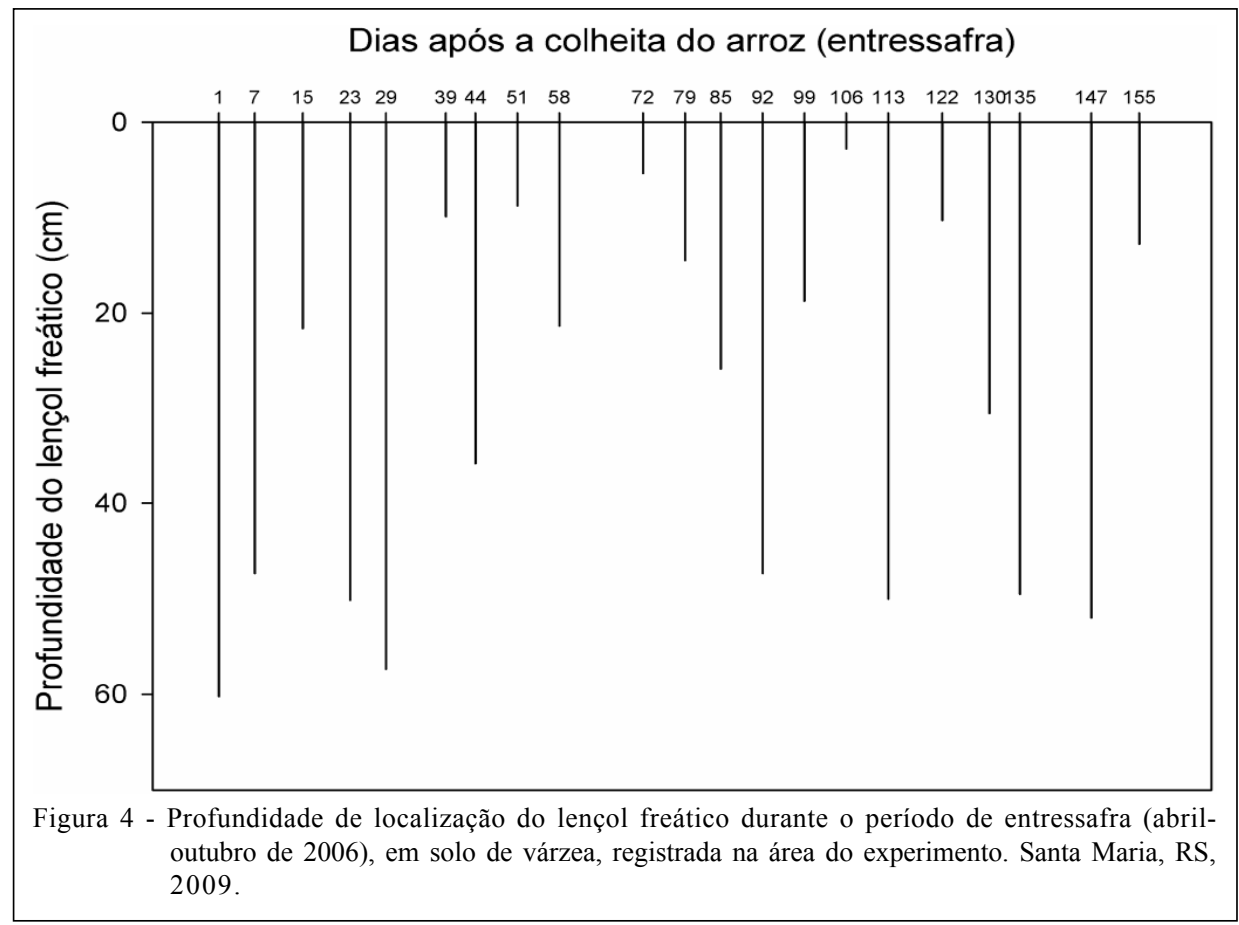

degradação do herbicida no PD, nas camadas mais profundas do solo.

\section{CONCLUSÕES}

Imazethapyr lixivia até $20 \mathrm{~cm}$ em solo de várzea, independentemente do sistema de cultivo. Ocorre maior concentração de imazethapyr na superfície do solo $(0-5 \mathrm{~cm})$, no sistema convencional, quando comparado com o sistema de plantio direto.

A quantidade total de herbicida remanescente no solo 540 dias após a última aplicação não é afetada pelo sistema de preparo do solo. Porém, o preparo de solo afeta a distribuição do imazethapyr no perfil. No sistema plantio convencional, o herbicida distribui-se de modo uniforme nos primeiros $15 \mathrm{~cm}$ de solo e, no sistema de plantio direto, apresenta menor concentração na camada de $0-5 \mathrm{~cm}$ e acumula-se entre $5-15 \mathrm{~cm}$ de profundidade.

\section{AGRADECIMENTOS}

Ao Conselho Nacional de Desenvolvimento Científico e Tecnológico $(\mathrm{CNPq})$, pelo auxílio financeiro e pela bolsa de iniciação científica. Ao INTA-Argentina, pelo financiamento dos estudos do primeiro autor.

\section{REFERÊNCIAS}

BASHAM, G.W.; LAVY, T.L. Microbial and photolytic dissipation of imazaquin in soil. Weed Science, v.35, p.865$870,1987$.
BATTAGLIN, W.A. et al. Occurrence of sulfonylurea, sulphonamide, imidazolinone, and other herbicides in rivers, reservoirs and ground water in the Midwestern United States, 1998. Science Total Environmental, v.248, p.123-133, 2000. Disponível em: <http://www.sciencedirect.com/ science?_ob=ArticleURL\&_udi=B6V78-4007 P 8 H 7\&_user $=\overline{687358 \& \text { rdoc }}=1 \&$ \&mt $=$ \&_orig $=$ search \&_sort $=\mathrm{d} \&$ $\mathrm{vie} w=\mathrm{c} \&$ a c c t $=\mathrm{C} 00 \overline{0} 037 \overline{8} 99 \&$ \&_vers i on $=1$ \& u r l V e r s i o n $=0$ \& u s e r i d = 6873 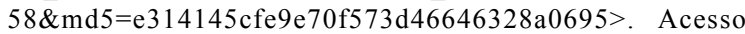 em: 26 de mar. 2009. Doi:10.1016/S0048-9697(99)00536-7.

BAUGHMAN, T.A.; SHAW, D.R. Effect of wetting/drying cycles on dissipation patterns of bioavailability imazaquin. Weed Science, v.44, n.2, p.380-382, 1996.

BÖRJESSON, E. et al. The fate of imazapyr in a Swedish railway embankment. Pest Management Science, v. 60, n. 6, p.544549, 2004. Disponível em: <http://www3.interscience.wiley.com/ journal $/ 108564464 /$ abstract?CRETRY $=1 \&$ SRETRY $=0>$. Acesso em: 20 de mar.2009. Doi: 10.1002/ps.864.

CURRAN, W.S. et al. Effect of tillage and application method on clomazone, imazaquim, and imazethapyr persistence. Weed Science, v.40, p.482-489, 1992.

FIRMINO, L.E. et al. Movimento do herbicida imazapyr no perfil de solos tropicáis. Revista Planta Daninha, v.26, n.1, p.223-230, 2008. Disponível em: <http://www.scielo.br/ s c i e lo.php ? s cript $=$ sci_art text \& pid $=$ S $0100-$ $83582008000100023 \& \operatorname{lng}=\mathrm{pt} \& \mathrm{nrm}=\mathrm{iso} \& \mathrm{t} \operatorname{lng}=\mathrm{pt}>$. Acesso em: 20 mar. 2009. doi:10.1590/S0100-83582008000100023.

FLINT, J.L.; WITT, W.W. Microbial degradation of imazaquin and imazethapyr. Weed Science, v.45, p.586-591, 1997. 
FRANCHINI, J.C. et al. Microbiological parameters as indicators of soil quality under various soil management and crop rotation systems in southern Brazil. Soil Tillage Research, v.92, p.18-29, 2007. Disponível em: <http:/www.sciencedirect.com/ science?_ob=ArticleURL\&_udi=B6TC6-4JB9MN8$1 \&$ \&user $=687358 \&$ rdoc $=1 \&_{-}$fmt $=\&$ \&_orig $=$ search $\&$ sort $=\mathrm{d} \&$ view $=$ c \&_ac ct $=$ C $000037899 \&$ _versio

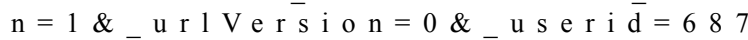
$358 \&$ md5 $=0 \mathrm{c} 40 \mathrm{~b} 7 \mathrm{baec} 3 \mathrm{c} 0978637 \mathrm{a} 80 \mathrm{ee} 1 \mathrm{daf0a} 72>$. Acesso em: 26 de mar. 2009. Doi:10.1016/j.still.2005.12.010

GONÇALVES, F.F. Estudo de métodos empregando HPLCDAD: Estudo de métodos empregando hplc-dad e lc-ms/ms para a determinação de resíduos de herbicidas em água e solo do cultivo de arroz irrigado. 2003. 170f. Tese (Doutorado em Química) - UFSM, Santa Maria, RS. Disponível em: http:// cascavel.cpd.ufsm.br/tede/tde_busca/arquivo.php?codArquivo=1715. Acesso: em 26 mar. 2009.

HOLLAWAY, K.L. et al. Persistence and leaching of imazethapyr and flumetsulam herbicides over a 4-year period in the highly alkaline soils of south-eastern Australia. Australian Journal of Experimental Agriculture, v.46, n.5, p.669-674, 2006. Disponível em: <http://www.publish.csiro.au/?paper=EA04223>. Acesso em: 20 de mar. 2009. Doi: 10.1071/EA04223.

JOHNSON, D.H. et al. Time-dependent adsorption of imazethapyr to soil. Weed Science, v.48, n.6, p.769-775, 2000. Disponível em: <http://www.bioone.org/toc/wees/48/6>. Acesso em: 26 de mar. 2009. Doi: 10.1614/00431745(2000)048[0769:TDAOIT]2.0.CO;2

JOURDAN, S.W. et al. Imazethapyr bioactivity and movement in soil. Weed Science, v.46, p.608-613, 1998.

MADANI, M.E. et al. pH effect and kinetic studies of the binding behaviour of imazethapyr herbicide on some Moroccan soils. Fresenius Environmental Bulletin, v.12, p.11141119,2003

MASSONI, P.F.S. et al. Controle de arroz vermelho em arroz tolerante a Imidazolinonas e o residual em genótipo de arroz não-tolerante. In: CONGRESSO BRASILEIRO DE ARROZ IRRIGADO, 5., 2007, Pelotas, RS. Anais... Pelotas: SOSBAI, 2007. V.2, p.230-233.

McDOWELL, R.W. et al. Dissipation of imazapyr, flumetsulam and thifensulfuron in soil. Weed Research, v.37, p.381-389, 1997.

MONKS D.C.; BANKS P.A. Effect of straw, ash, and tillage on dissipation of imazaquim and imazetapyr. Weed Science, v.41, 133-137, 1993.

ONA, S. et al. Sorption and predicted mobility of herbicides in Baltic soils. Environmental Science Health, Part B, v.42, n.6, p.641-647, 2007.
RENNER, K.A. et al. Effect of tillage and application method on corn (Zea mays) response to imidazolinone residues in soil. Weed Technololgy, v.12, n.2, 281-285, 1998.

SEIFERT, S. et al. Imazaquim mobility and persistence in a Sarkey Clay soil as influenced by tillage systems. Weed Science, v.49, p.571-577, 2001. Disponível em: >http:// w w w. bioon e.org/doi/ful1/10.1614/00431745\%282001\%29049\%5B0571\%3AIMAPIA\%5D2.0.CO\%3B2>. Acesso em 29 Mar. 2009. Doi: 10.1614/00431745(2001)049[0571:IMAPIA]2.0.CO;2

SHANER, D.L.; O'CONNOR, S. Eds. Imidazolinones Herbicides. Boca Raton: CRC, 1991. 290p.

TAN, S. et al. Imidazolinone-tolerant crops: history, current status and future. Pest Management Science, v.61, n.3, p.246257, 2005. Disponível em: <http://www3.interscience.wiley.com/ journal $/ 109861207 /$ abstract?CRETRY $=1 \&$ SRETRY $=0>$. Acesso em: 20 mar. 2009. Doi:10.1002/ps.993.

TAN, S. et al. Herbicidal inhibitors of amino acid biosynthesis and herbicide-tolerant crops. Amino Acids, v.30, p.195-204, 2006. Disponível em: <http://www.springerlink.com/content/ f0748891g4111670>. Acesso em: 29 Mar. 2009. Doi: 10.1007/ s00726-005-0254-1

ULBRICH, A.V. et al. Persistence and carryover effect of Imazapic and Imazapyr in Brazilian cropping systems. Weed Technology, v.19, p.986-991, 2005. Disponível em: <http:// www.bioone.org/doi/full/10.1614/WT-04-208R2.1>. Acesso em: 29 Mar. 2009. Doi: 10.1614/WT-04-208R2.1.

VIZZOTTO, V.R. et al. Efeito do pisoteio bovino em algumas propriedades físicas do solo de várzea. Ciência Rural, v.30, n.6, p. 965-969, 2000. Disponível em: <http://www.scielo.br/ s c i e lo.php? s cript $=$ sci_arttext \& pid = S $0103-$ $84782000000600007 \& \operatorname{lng}=\mathrm{en} \& \mathrm{nrm}=\mathrm{iso} \& \mathrm{t} \operatorname{lng}=\mathrm{pt}>$. Acesso em: 29 Mar. 2009. Doi: 10.1590/S0103-84782000000600007.

VAN WYK, L.J.; REINHARDT, C.F. A bioassay technique detects imazethapyr leaching and liming-dependent activity. Weed Science, v.15, n.3, p.1-6, 2001.

WILLIAMS, B.J. et al. Weed management systems for Clearfield rice. Louisiana Agriculture Chemistry, v.45, n.3, p.16$17,2002$.

ZHANG, W. et al. Effect of rotational crop herbicides on water- and dry-seeded Oryza sativa. Weed Science, v.48, p.755-760, 2000. Disponível em: <http://www.bioone.org/doi/abs/10.1614/00431745(2000)048\%5B0755\%3AEORCHO\%5D2.0.CO\%3B2>. Acesso em: 29 Mar. 2009. Doi: 10.1614/00431745(2000)048[0755:EORCHO]2.0.CO;2. 